\title{
Electrophoretic Mobility of a Concentrated Suspension of Spherical Particles
}

\author{
Eric Lee, Jhih-Wei Chu, and Jyh-Ping Hsu ${ }^{1}$ \\ Department of Chemical Engineering, National Taiwan University, Taipei, Taiwan 10617, Republic of China
}

Received July 17, 1998; accepted September 23, 1998

\begin{abstract}
The electrophoretic behavior of concentrated spherical colloidal particles is analyzed theoretically for all levels of scaled surface potential $\phi_{a}$, taking the effect of double-layer polarization (DLP) into account. The result of numerical simulation reveals that for a very small $\kappa a(<0.01)$, $\kappa$ and $a$ being, respectively, the reciprocal Debye length and the particle radius, or a very large к $a(>100)$, using a linearized Poisson-Boltzmann equation (PBE) and neglecting the effect of DLP is reasonable; for an intermediate $\kappa a$, appreciable deviation may result. The deviation is negative if $\kappa a$ is small, and positive if $\kappa a$ is large. The mobility against $\kappa a$ curve may have a local minimum and a local maximum. If $\phi_{a}$ is low, the mobility increases with the porosity of the system under consideration, and for a fixed porosity, the mobility increases with $\kappa a$. If $\phi_{a}$ is high and $\kappa a$ is small, the effect of $\phi_{a}$ (i.e., solving a nonlinear PBE ) on the mobility of a particle is more significant than that of double-layer polarization, and the reverse is true if $\kappa a$ is large. For an intermediate $\kappa a$, the effect of DLP is more significant than that of $\phi_{a}$ when the porosity is high, and the reverse is true if it is low. $\odot 1999$ A cademic Press
\end{abstract}

Key Words: electrophoretic mobility; double-layer polarization, concentrated suspension; spherical particles.

\section{INTRODUCTION}

Electrophoresis is one of the electrokinetic phenomena which has various applications in practice. Typical example includes separation of proteins and stabilization of soft soil. The phenomenon is governed by the so-called electrokinetic equations, which comprise the equation that describes the conservation of ions, the Poisson-Boltzmann equation, and the Navier-Stokes equation. The second describes the spatial variation of the electrical potential, and the last the flow field of the system under consideration. In general, these equations are nonlinear and coupled with each other, which renders them extremely difficult to solve analytically. Often, it is assumed that the electrical potential is low and the electrical double layer around a charged entity is either very thick or very thin. Under these conditions, the Poisson-Boltzmann equation can be linearized and decoupled from the Navier-Stokes equation

\footnotetext{
${ }^{1}$ To whom correspondence should be addressed. E-mail: t8504009@ccms. ntu.edu.tw.
}

$(1-4,7-9)$. Otherwise, the electrokinetic equations needed to be solved numerically $(5,6)$. Previous investigations are focused mainly on the behavior of an isolated particle in an infinite solution (1-9). In other words, the presence of other objects, such as the rigid boundary and nearby entities, is assumed to have a negligible influence on its behavior. By adopting a unit cell model, Levine and Neale (10) derived the electrophoretic mobility of a system containing multiple spheres under the condition of low electrical potential, which is valid for an arbitrary thickness of the double layer. They concluded that, due to the overlapping of the electrical double layers, the mobility decreases rapidly with the increase in the thickness of a double layer and/or the decrease in the porosity of the system. The same problem was also discussed by Kozak and Davis $(11,12)$ and by Ohshima (13). In the former, an arbitrary level of electrical potential was considered, taking the effect of double-layer polarization (DLP), the distortion of the double layer near a charged entity due to its relative motion with respect to the surrounding liquid medium as a response to the applied electric field, into account, but the overlapping of the double layers between two nearby particles was neglected. In the latter, an approximate expression for mobility was derived in which the complicated numerical integration involved in Levine and Neale (10) was avoided. The approximate expression was found to have a relative error less than $4 \%$.

In the present work, the electrophoresis of concentrated spherical colloidal particles is analyzed theoretically for all levels of electrical potential, taking the effect of DLP, which was neglected in most of the aforementioned studies, into account. In this case the general electrokinetic equations needed to be solved simultaneously. The idealized model adopted by Zydney (14) is used in conjunction with the pseudo-spectral discretization scheme proposed in our previous study for the case of a sphere in a spherical cavity $(15,16)$.

\section{THEORY}

We consider identical, non-conducting, spherical particles of radius $a$ in a $z_{1}: z_{2}$ electrolyte solution, $z_{1}$ and $z_{2}$ being, respectively, the valences of cations and anions, and $\alpha=$ 


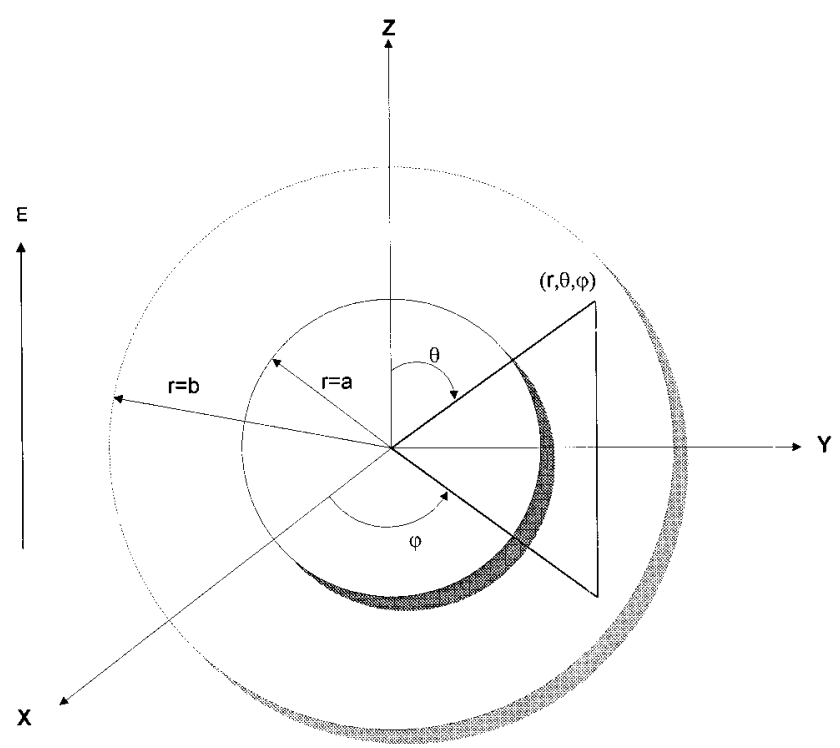

FIG. 1. Schematic representation of the system under consideration.

$-z_{2} / z_{1}$. The electroneutrality in the bulk liquid phase requires that $n_{20}=\left(n_{10} / \alpha\right), n_{10}$ and $n_{20}$ being the bulk concentrations of cations and anions, respectively. The unit cell model proposed by Levine and Neale (10) is used. Referring to Fig. 1, each sphere is surrounded by a concentric spherical shell of liquid phase of radius $b$. The spherical coordinates $(r, \theta, \varphi)$, with origin at the center of the particle, are adopted in the following analysis. A uniform electric field $E$ is applied in the $z$-direction $(\theta=0)$. Suppose that the liquid phase is incompressible and has constant physical properties. Also, the motion of particles is slow so that the system under consideration is at a quasi-steady state. Under these conditions, the electrophoretic motion of the particle is governed by the following set of three equations.

\section{Conservation of Ions}

The conservation of ions leads to

$$
\vec{\nabla} \cdot\left[D_{j}\left[\vec{\nabla} n_{j}+\frac{z_{j} e n_{j}}{k T} \vec{\nabla} \phi\right]+n_{j} \vec{\nabla}\right]=0, \quad j=1,2,
$$

where $\vec{\nabla}$ denotes the gradient operator, $e$ and $\phi$ are, respectively, the elementary charge and the electrical potential, $\vec{v}$ represents the fluid velocity, $k$ and $T$ are the Boltzmann constant and the absolute temperature, respectively, and $n_{j}, D_{j}$, and $z_{j}$ are the number concentration, the diffusivity, and the valence of ion species $j$, respectively.

\section{Electrical Potential}

Here, we assume that the time scale for double-layer relaxation is much smaller than that of the motion of a particle, and, therefore, the spatial variation of the electrical potential can be described by the Poisson-Boltzmann equation

$$
\nabla^{2} \phi=-\frac{\rho}{\epsilon}=-\sum_{j=1}^{2} \frac{z_{j} e n_{j}}{\epsilon}
$$

where $\epsilon$ and $\rho$ denote the permittivity of the liquid phase and the spatial charge density, respectively.

\section{Flow Field}

The flow field is described by the Navier-Stokes equation in the creeping flow region

$$
\begin{gathered}
\vec{\nabla} \cdot \overrightarrow{\mathrm{v}}=0 \\
\eta \nabla^{2} \overrightarrow{\mathrm{v}}-\vec{\nabla} p-\rho \vec{\nabla} \phi=0,
\end{gathered}
$$

where $p, \eta$, and $\rho$ denote, respectively, the pressure, the viscosity, and the density of fluid.

Suppose that $\phi$ can be decomposed as (16)

$$
\phi=\phi_{1}+\phi_{2}
$$

where $\phi_{1}$ and $\phi_{2}$ represent, respectively, the electrical potential that would exist in the absence of the applied electric field and that outside the particle arising from the applied electric field. The effect of DLP is taken into account by considering the expression (6)

$$
n_{j}=n_{j 0} \exp \left(-\frac{z_{j} e\left(\phi_{1}+\phi_{2}+g_{j}\right)}{k T}\right), \quad j=1,2,
$$

where $g_{j}$ denotes a perturbation function for potential which accounts for the effects of fluid flow on concentration. If $\phi_{2}$ and $g_{j}$ are small compared to both $k T / e$ and $\phi_{1}$, Eq. [6] can be linearized as

$$
n_{j}=n_{j 0} \exp \left(-\frac{z_{j} e \zeta_{a}}{k T} \phi_{1}^{*}\right)\left[1-\frac{z_{j} e \zeta_{a}}{k T}\left(\phi_{2}^{*}+g_{j}^{*}\right)\right], \quad j=1,2
$$

where $\phi_{j}^{*}=\phi_{j} / \zeta_{a}, g_{j}^{*}=g_{j} / \zeta_{a}$, and the surface potential of a particle is characterized by the zeta potential $\zeta_{a}$. It should be pointed out that the fact that $\phi_{2}$ and $g_{j}$ are small does not imply that their effects are insignificant (6).

For convenience, scaled properties are used in the following analyses. Here, the radius of a particle $a$ is chosen as the characteristic length and the electrophoretic velocity of an isolated particle in an electric field of strength $\left(\zeta_{a} / a\right)$ based on the Smoluchowski's theory $U_{E}\left(=\epsilon \zeta_{a}^{2} / \eta a\right)$ as the characteristic velocity. Instead of solving Eqs. [3] and [4] directly for 
velocity and pressure, the governing equation which describes the stream function $\psi$, obtained by taking the curl of Eq. [4] subject to Eq. [6], is solved. The $r$ - and $\theta$-components of velocity $\mathrm{V}, \mathrm{v}_{r}$, and $\mathrm{v}_{\theta}$, can be evaluated from $\psi$ by $\mathrm{v}_{r}=$ $-(\partial \psi / \partial \theta) / r^{2} \sin \theta$ and $\mathrm{v}_{\theta}=(\partial \psi / \partial r) / r \sin \theta$, respectively. For convenience, a scaled stream potential $\psi^{*}=\psi / U_{E} a^{2}$ is defined.

The symmetric nature of the spherical coordinates adopted suggests that the problem under consideration can be reduced to a one-dimensional one (16); i.e., $\phi_{2}^{*}, g_{1}^{*}, g_{2}^{*}$, and $\psi^{*}$ can be written as $\phi_{2}^{*}=\Phi_{2}(r) \cos \theta, g_{1}^{*}=G_{1}(r) \cos \theta, g_{2}^{*}=$ $G_{2}(r) \cos \theta$, and $\psi^{*}=\Psi(r) \sin ^{2} \theta$. The governing equations, Eqs. [1]-[4], are then simplified under the above statements. It can be shown that the variation of $\phi_{1}^{*}$, the scaled electric potential at equilibrium, is governed by

$$
L \phi_{1}^{*}=-\frac{1}{(1+\alpha)} \frac{(\kappa a)^{2}}{\phi_{r}}\left[\exp \left(-\phi_{\mathrm{r}} \phi_{1}^{*}\right)-\exp \left(\alpha \phi_{\mathrm{r}} \phi_{1}^{*}\right)\right]
$$

where the scaled surface potential $\phi_{r}$, the operator $L$, and the Debye-Huckel parameter $\kappa$ are defined, respectively, by

$$
\begin{aligned}
\phi_{r} & =\zeta_{a} z_{1} e / k T \\
L & \equiv \frac{d^{2}}{d r^{* 2}}+\frac{2}{r^{*}} \frac{d}{d r^{*}}-\frac{2}{r^{* 2}},
\end{aligned}
$$

where $r^{*}=r / a$, and

$$
\kappa=\left[\sum_{j=1}^{2} n_{j 0}\left(e z_{j}\right)^{2} / \epsilon k T\right]^{1 / 2}
$$

The boundary conditions associated with Eq. [8] are

$$
\begin{gathered}
\phi_{1}^{*}=1 \quad \text { at } r^{*}=1 \\
\frac{d \phi_{1}^{*}}{d r^{*}}=0 \quad \text { at } r^{*}=b / a,
\end{gathered}
$$

where the second condition implies that the unit cell as a whole is electrically neutral, and there is no current flow between adjacent cells. Equation [8] needs to be solved simultaneously with the equations (16)

$$
\begin{gathered}
{\left[L-\frac{(\kappa a)^{2}}{1+\alpha}\left[\exp \left(-\phi_{r} \phi_{1}^{*}\right)+\alpha \exp \left(\alpha \phi_{r} \phi_{1}^{*}\right)\right]\right] \Phi_{2}} \\
=\frac{(\kappa a)^{2}}{1+\alpha}\left[G_{1} \exp \left(-\phi_{r} \phi_{1}^{*}\right)+\alpha \mathrm{G}_{2} \exp \left(\alpha \phi_{r} \phi_{1}^{*}\right)\right] \\
L G_{1}-\phi_{r} \frac{d \phi_{1}^{*}}{d r^{*}} \frac{d G_{1}}{d r^{*}}=\mathrm{Pe}_{1} \mathrm{~V}_{r}^{*} \frac{d \phi_{1}^{*}}{d r^{*}}
\end{gathered}
$$

$$
\begin{array}{r}
L G_{2}+\alpha \phi_{r} \frac{d \phi_{1}^{*}}{d r^{*}} \frac{d G_{2}}{d r^{*}}=\mathrm{Pe}_{2} \mathrm{~V}_{r}^{*} \frac{d \phi_{1}^{*}}{d r^{*}} \\
D^{4} \Psi=-\frac{(\kappa a)^{2}}{1+\alpha}\left[\left(n_{1}^{*} G_{1}+\alpha n_{2}^{*} G_{2}\right) \frac{d \phi_{1}^{*}}{d r^{*}}\right],
\end{array}
$$

where $\mathrm{Pe}_{j}=\epsilon\left(Z_{1} e / k T\right)^{2} / \eta D_{j}, j=1,2$, is the electric Peclet number of ion species $j, n_{1}^{*}=\exp \left(-\phi_{r} \phi_{1}^{*}\right), n_{2}^{*}=$ $\exp \left(\alpha \phi_{r} \phi_{1}^{*}\right)$, and the operator $D^{4}$ is defined as

$$
D^{4}=\left(D^{2}\right)^{2}=\left(\frac{d^{2}}{d r^{* 2}}-\frac{2}{r^{* 2}}\right)^{2}
$$

The boundary conditions associated with Eqs. [9]-[12] are

$$
\begin{aligned}
& \frac{d \Phi_{2}}{d r^{*}}=0 \quad \text { at } r^{*}=1 \\
& \frac{d \Phi_{2}}{d r^{*}}=-E_{z}^{*} \quad \text { at } r^{*}=b / a \\
& \frac{d G_{j}}{d r^{*}}=0 \quad \text { at } r^{*}=1, j=1,2 \\
& G_{j}=-\Phi_{2} \quad \text { at } r^{*}=b / a, j=1,2
\end{aligned}
$$

$\Psi=-\frac{1}{2} U^{*} r^{* 2} \quad$ and $\quad \frac{d \Psi}{d r^{*}}=-U^{*} r^{*} \quad$ at $r^{*}=1$

$\Psi=\left[\frac{1}{r^{*}} \frac{d^{2} \Psi}{d r^{* 2}}-\frac{2}{r^{* 3}}\right] \Psi=0 \quad$ at $r^{*}=b / a$,

where $E_{z}^{*}=E_{z} a / \zeta_{a}$, and $U^{*}=U / U_{E}, E_{z}$ and $U$ being, respectively, the $z$-component of the electric field and the terminal velocity of a particle. In these expressions, Eq. [13a] states that the particle is non-conducting, Eq. [13b] specifies the applied electric field, Eq. [13c] implies that the particle surface is impermeable to fluid, Eq. [13d] suggests that ion species achieve the equilibrium value at the virtual surface, and Eq. [13e] states that the particle is moving with velocity $U$. Equation [13f] implies that the virtual surface is steady and satisfies Kuwabara's model of zero vorticity; this will lead to a result which is consistent with that predicted by the Smoluchowski's equation for the case of isolated particles (10).

The mobility of a particle can be evaluated on the basis of a force balance. At the steady state, the sum of the external forces acting on a particle in the $z$-direction, which includes the electrical force $F_{\mathrm{E} z}$ and the hydrodynamic force $F_{\mathrm{D} z}$, vanishes. It can be shown that (16) 


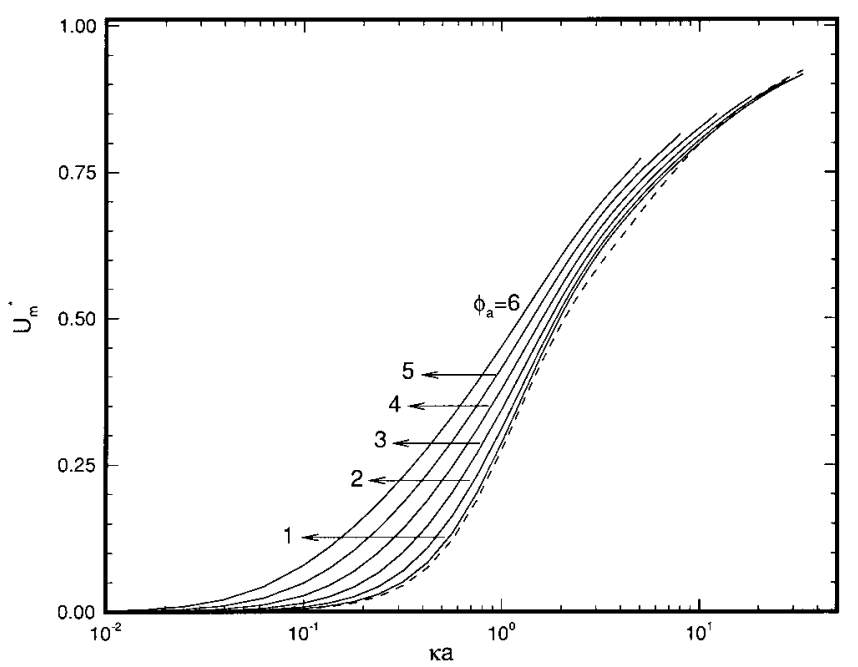

FIG. 2. Variation of scaled mobility $U_{m}^{*}$ as a function of $\kappa a$ at various levels of scaled surface potential $\phi_{a}$. Key: $H=0.5 ; a=1 ; b=2$; (---) result of Ohshima (13).

$$
\begin{gathered}
F_{\mathrm{E} z}=\frac{8}{3} \pi \epsilon \zeta_{a}^{2}\left(r^{*} \frac{d \phi_{1}^{*}}{d r^{*}} \Phi_{2}\right)_{r^{*}=1}=F_{\mathrm{E} z}^{*} \pi \epsilon \zeta_{a}^{2} \\
F_{\mathrm{D} z}=\frac{4}{3} \pi \epsilon \zeta_{a}^{2}\left(r^{* 4} \frac{\partial}{\partial r^{*}}\left(\frac{D^{2} \Psi}{r^{* 2}}\right)\right)_{r^{*}=1}+\frac{4}{3} \pi \epsilon \zeta_{a}^{2} \frac{(\kappa a)^{2}}{(1+\alpha a) \phi_{r}} \\
\times\left[r^{* 2}\left[\exp \left(-\phi_{r} \phi_{1}^{*}\right)-\exp \left(\alpha \phi_{r} \phi_{1}^{*}\right)\right] \Phi_{2}\right]_{r^{*}=1} .
\end{gathered}
$$

According to O'Brien and White (6), the problem of solving Eqs. [8]-[13f] can be decomposed into two sub-problems. In the first problem a particle moves at a uniform velocity $U$ in the absence of the applied field, and in the second problem, the particle is held fixed in the applied electric field. The force required to move the particle in the first problem, $f_{1}$, can be expressed as $f_{1}=\delta U^{*}$, and the force exerts on the particle in the second problem, $f_{2}$, as $f_{2}=\beta E_{z}^{*}$. Therefore, we have $U_{m}^{*}$ $=U^{*} / E_{z}^{*}=-\beta / \delta, U_{m}^{*}$ being the scaled mobility of the particle. Here, $\delta$ denotes the force per unit velocity exerting on a particle in the absence of electric field, and $-\beta$ is the sum of the scaled electric force in the $z$-direction, $F_{\mathrm{E} z}^{*}\left(=F_{\mathrm{Ez}} / \pi \epsilon \zeta_{a}^{2}\right)$, and the scaled drag force in the $z$-direction, $F_{\mathrm{D} z}^{*}\left(=F_{\mathrm{D} z} / \pi \epsilon \zeta_{a}^{2}\right)$. Details about the derivations of the governing equations, calculation of mobility, and the numerical scheme employed can be found in Lee et al. (16).

\section{RESULTS AND DISCUSSION}

Figure 2 shows the variation of the scaled mobility $U_{m}^{*}$ as a function of $\kappa a$ at various levels of scaled surface potential $\phi_{a}$ for the case in which the effect of DLP is neglected. The result of Ohshima (13), which was based on a linearized Poisson-Boltzmann equation (LPBE) and which neglected the effect of DLP, is also presented for comparison. For convenience, Ohshima's model is abbreviated hereafter as
OM. As can be seen from Fig. 2, $U_{m}^{*}$ increases monotonically with $\kappa a$. For fixed particle size, this implies that the thicker the electrical double layer (smaller $\kappa$ ), or the more significant the electrical interaction between two particles, the smaller the mobility. This is consistent with the predictions of Levine and Neale (10). Figure 2 suggests that if DLP is neglected, using LPBE will underestimate the mobility, in general. The deviation becomes insignificant, however, if $\kappa a$ is either very small or very large. A comparison of the present result with that of OM reveals that the effects of DLP and relaxation are reflected by the terms involving $\kappa a$ in Eqs. [9] and [12]. If $\kappa a$ is small, it reduces to the corresponding expression of OM, and the effect of DLP becomes negligible.

The variations of the scaled mobility $U_{m}^{*}$ as a function of $\kappa a$ at various levels of scaled surface potential $\phi_{a}$ for the case in which the effect of DLP is taken into account are illustrated in Figs. 3 and 4 for two different $H(=a / b)$. The result based on $\mathrm{OM}$ is also presented in these figures for comparison. Here, due to the nature of the pseudo-spectral method adopted, the numerical procedure becomes inefficient if $\kappa a$ exceeds a critical value, which depends largely on the magnitudes of $H$ and $\phi_{r}$. Nevertheless, the general trend can still be predicted. Figures 3 and 4 reveal that, for a very small $(<0.01)$ or a very large $(>100) \kappa a$, OM is satisfactory. For an intermediate $\kappa a$, depending upon the magnitudes of $\kappa a, H$, and $\phi_{a}$, OM may either underestimate or overestimate $U_{m}^{*}$. The deviation depends largely upon two competing factors. First, as pointed out previously, using LPBE will underestimate $U_{m}^{*}$. Second, according to Kozak and Davis $(11,12)$, the electric field induced by a charged particle has the effect of lowering the applied electric field, and therefore, using LPBE will overestimate $U_{m}^{*}$. Note that, since double-layer interactionis neglected in their analysis, the conclusion is suited for large $\kappa a$ only. Also, for

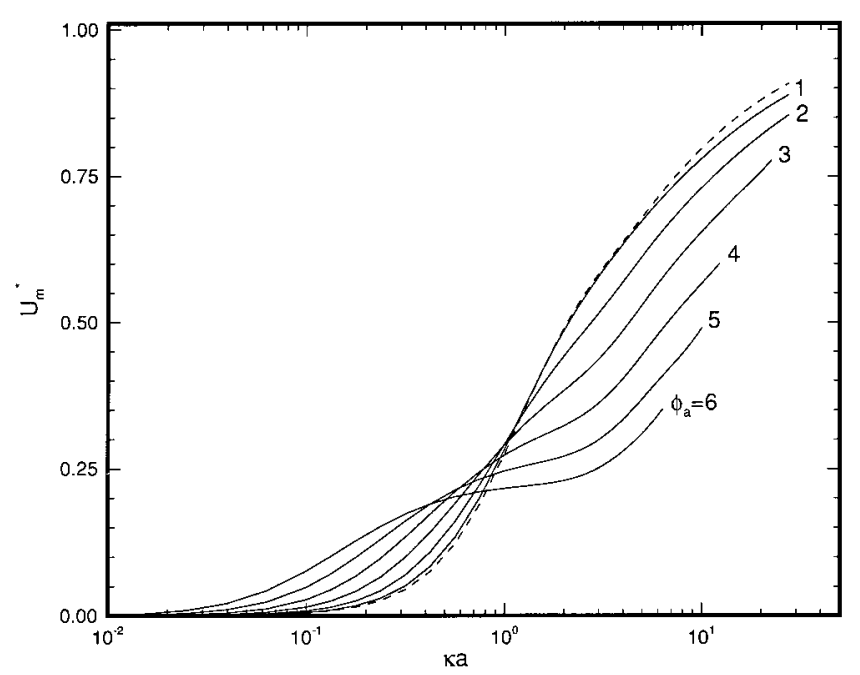

FIG. 3. Variation of scaled mobility $U_{m}^{*}$ as a function of $\kappa a$ at various levels of scaled surface potential $\phi_{a}$ for the case $\mathrm{Pe}_{1}=\mathrm{Pe}_{2}=0.1$ and $\alpha=1$. Key: same as Fig. 2. 


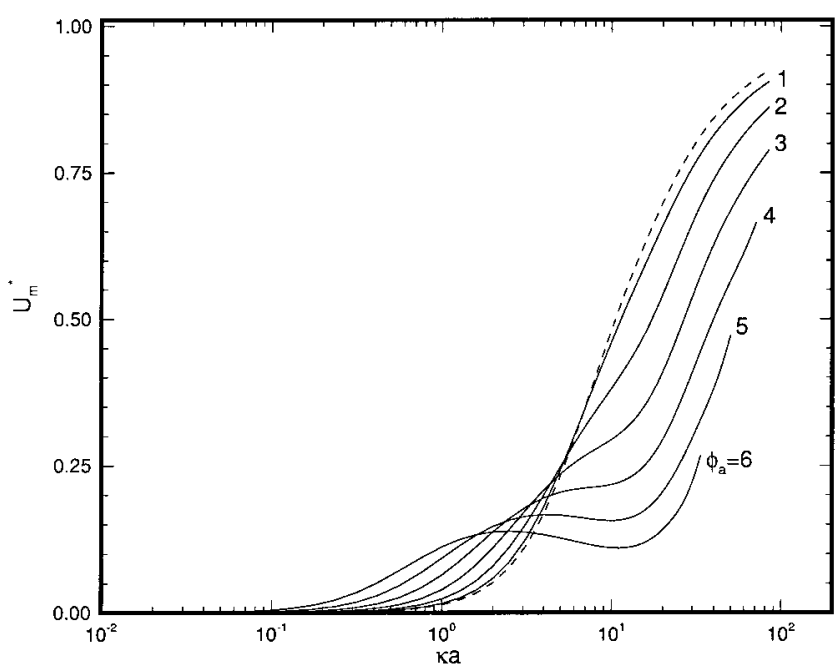

FIG. 4. Variation of scaled mobility $U_{m}^{*}$ as a function of $\kappa a$ at various levels of scaled surface potential $\phi_{a}$ for the case $\mathrm{Pe}_{1}=\mathrm{Pe}_{2}=0.1$ and $\alpha=1$. Key: same as Fig. 2 except that $H=0.833$.

fixed surface potential, the thinner the electrical double layer (larger $\kappa a$ ), the greater the gradient of potential, and, therefore, the stronger the electric field induced by a particle. On the other hand, referring to Fig. 2, the first factor is more pronounced for a smaller $\kappa a$. Therefore OM will underestimate $U_{m}^{*}$ if $\kappa a$ is small, and it will overestimate $U_{m}^{*}$ if $\kappa a$ is large. Note that according to Fig. 2, in the absence of the effect of DLP $U_{m}^{*}$ increases with $\kappa a$. On the other hand, if DLP is considered, since the electric field induced by a charged particle has the effect of lowering the applied electric field, the larger the $к \mathrm{a}$, the stronger the electric field induced, and therefore, the smaller the $U_{m}^{*}$. The net effect is that the variation of $U_{m}^{*}$ as a function of $\kappa a$ may have a local minimum and a local maximum, as can be seen from Fig. 4. A comparison between Figs. 3 and 4 reveals that if $H$ is large, or the concentration of particles is high, the local extreme can be observed at a lower surface potential.

Figures 5 and 6 show, respectively, the variations of the scaled electric force in the $z$-direction $F_{\mathrm{E} z}^{*}$ and the parameter $-\beta$ as a function of $\kappa a$ at various levels of scaled surface potential $\phi_{a}$. Figures 3, 5, and 6 reveal that the variations of $U_{m}^{*}$ and $F_{\mathrm{E} z}^{*}$ have the same trend. Since $U_{m}^{*}=-\beta / \delta$ and $-\beta$ $=F_{\mathrm{E} z}^{*}+F_{\mathrm{D} z}^{*}$, the qualitative behavior of $U_{m}^{*}$ is mainly determined by $-\beta$, and $\delta$ has a quantitative effect on $U_{m}^{*}$ only. This is similar to the case of a particle in a cavity (16).

The variations of the scaled mobility $U_{m}^{*}$ as a function of the porosity of the system under consideration, defined by [1 $\left.(a / b)^{3}\right]$, at various values of $\kappa a$ for two levels of surface potential $\phi_{a}$ are illustrated in Figs. 7 and 8. The corresponding results predicted by $\mathrm{OM}$ are also shown for comparison. Note that if the porosity becomes unity, the system reduces to isolated particles. Figure 7 suggests that if $\phi_{a}$ is low, $U_{m}^{*}$ increases with porosity, and for a fixed porosity, $U_{m}^{*}$ increases with $\kappa a$. The deviation of $\mathrm{OM}$ from the present model is

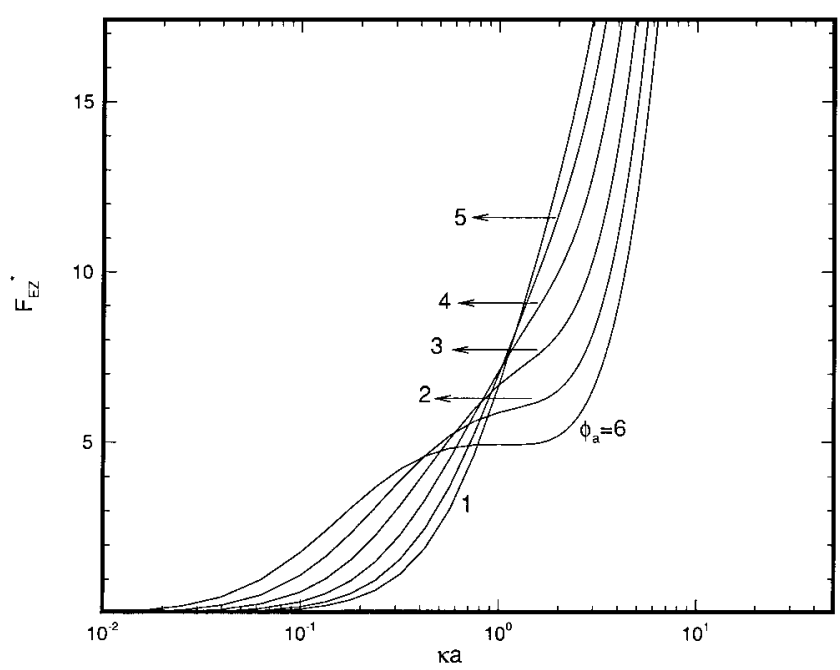

FIG. 5. Variation of scaled force $F_{\mathrm{E} z}^{*}$ as a function of $\kappa a$ at various levels of scaled surface potentials $\phi_{a}$ for the case $\mathrm{Pe}_{1}=\mathrm{Pe}_{2}=0.1, \alpha=1, H=0.5$, $a=1$, and $b=2$.

inappreciable. As suggested by Fig. 8, however, it becomes significant at a higher $\phi_{a}$. In this case, if $\kappa a$ is small $(<0.1)$, the effect of $\phi_{a}$ (i.e., solving a nonlinear Poisson-Boltzmann equation) on the mobility of a particle is more significant than that of DLP, and OM will underestimate $U_{m}^{*}$. On the other hand, if $\kappa a$ is large $(>10)$, the reverse is true. For an intermediate $\kappa a$, the effect of DLP is more significant than that of $\phi_{a}$ when the porosity is high, and the reverse is true if it is low.

The variations of the scaled mobility $U_{m}^{*}$ as a function of $\kappa a$ at various values of $\alpha\left(=-z_{2} / z_{1}=-\right.$ valence of anions/ valence of cations) are shown in Figs. 9 and 10. Since $n_{2}^{*}=$ $\exp \left(\alpha \phi_{r} \phi_{1}^{*}\right)$, the extent of the variation of $n_{2}^{*}$ as the electrical potential changes varies with $\alpha$. If $\alpha>1$, increasing $\alpha$ has the effect of raising the absolute value of the second term in the

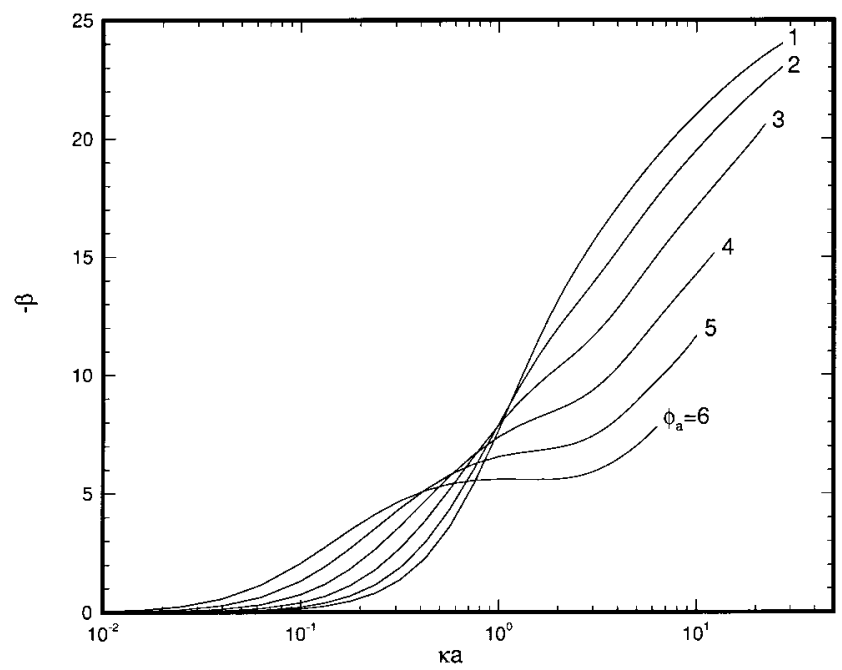

FIG. 6. Variation of parameter $-\beta$ as a function of $\kappa a$ at various levels of scaled surface potentials $\phi_{a}$ for the case in Fig. 5 . 


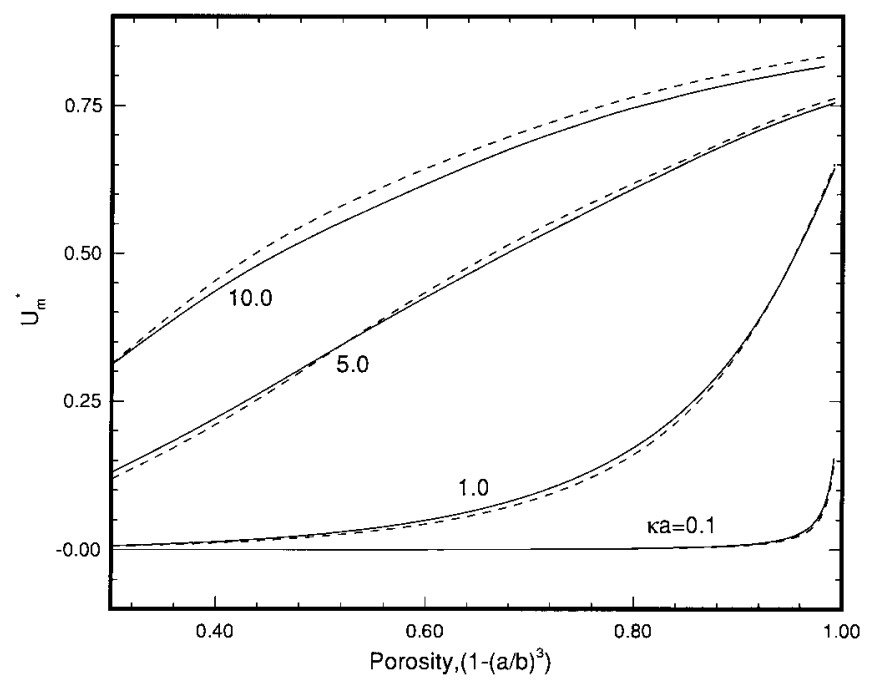

FIG. 7. Variation of scaled mobility $U_{m}^{*}$ as a function of porosity at various values of $\kappa a$ for the case $\mathrm{Pe}_{1}=\mathrm{Pe}_{2}=0.1, \phi_{a}=1$, and $\alpha=1$. Key: (---) result of Ohshima (13).

square bracket on the right-hand side of Eq. [7]. This is similar to the effect of increasing $\phi_{r}$. A comparison between Figs. 3 and 9 reveals that the effect of $\alpha$ on the qualitative behavior of the $U_{m}^{*}$ against $\kappa a$ curve is similar to that of $\phi_{a}$. Conclusions similar to those obtained from Fig. 9 can be drawn from Fig. 10 for the case in which $\alpha<1$.

The major difference between the present work and the relevant studies in the literature can be summarized as follows. In the analysis of Levine and Neale (10) the linearized Poisson-Boltzmann equation, i.e., the linearized version of Eq. [8], was considered. The effects of DLP and relaxation were neglected, but the overlapping of adjacent double layers was

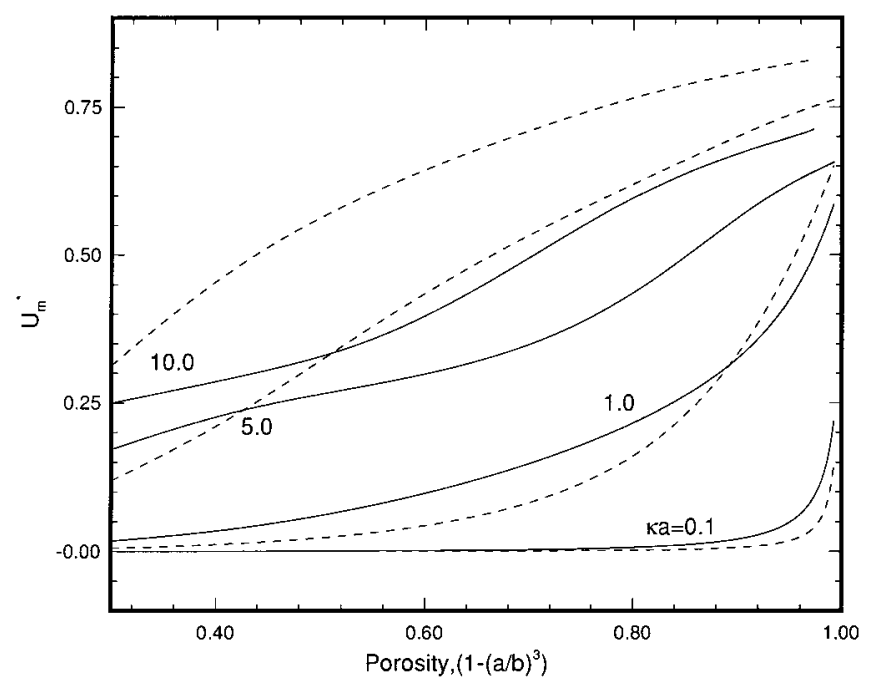

FIG. 8. Variation of scaled mobility $U_{m}^{*}$ as a function of porosity at various values of $\kappa a$ for the case $\mathrm{Pe}_{1}=\mathrm{Pe}_{2}=0.1, \phi_{a}=3$, and $\alpha=1$. Key: same as Fig. 7.

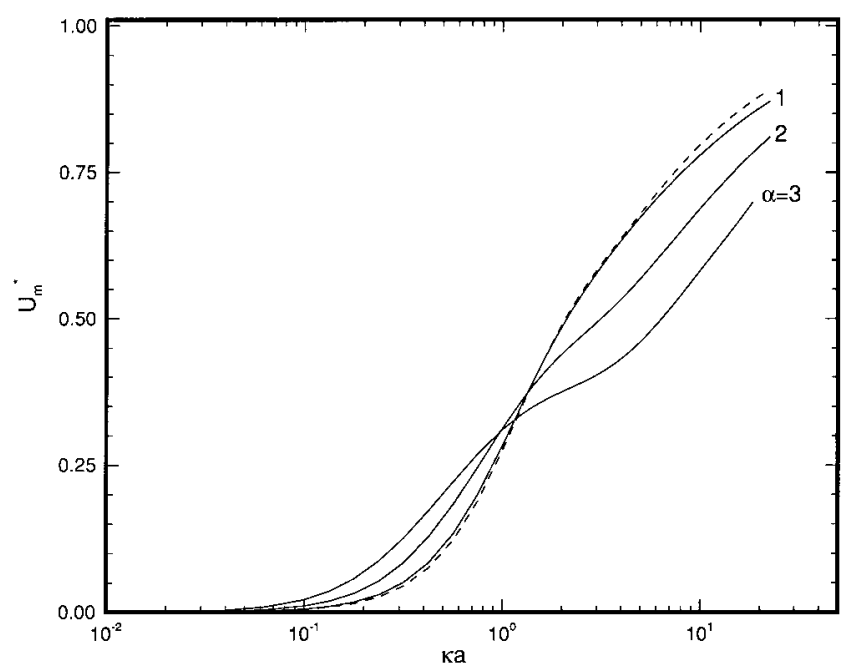

FIG. 9. Variation of scaled mobility $U_{m}^{*}$ as a function of $\kappa a$ at various values of $\alpha$ ( $=-$ valence of anions/valence of cations) for the case $\mathrm{Pe}_{1}=\mathrm{Pe}_{2}$ $=0.1$ and $\phi_{a}=1$. Key: same as Fig. 2 .

taking into account. That is, the right-hand side of Eq. [9] was assumed to be zero, and the boundary condition, Eq. [8e], was adopted. The effect of DLP was considered by Kozak and Davis (11). However, since the equilibrium potential within a cell was approximated by that of an isolated particle, Eq. [8e] was not considered, and therefore, the result obtained is best suited for the case of thin double layers; i.e., the interaction between adjacent double layers is insignificant. The analysis of Ohshima (13) is similar to that of Levine and Neale (10). The main difference between the two is that the former proposes an approximate method which is capable of simplifying the complicated exact solution of the latter.

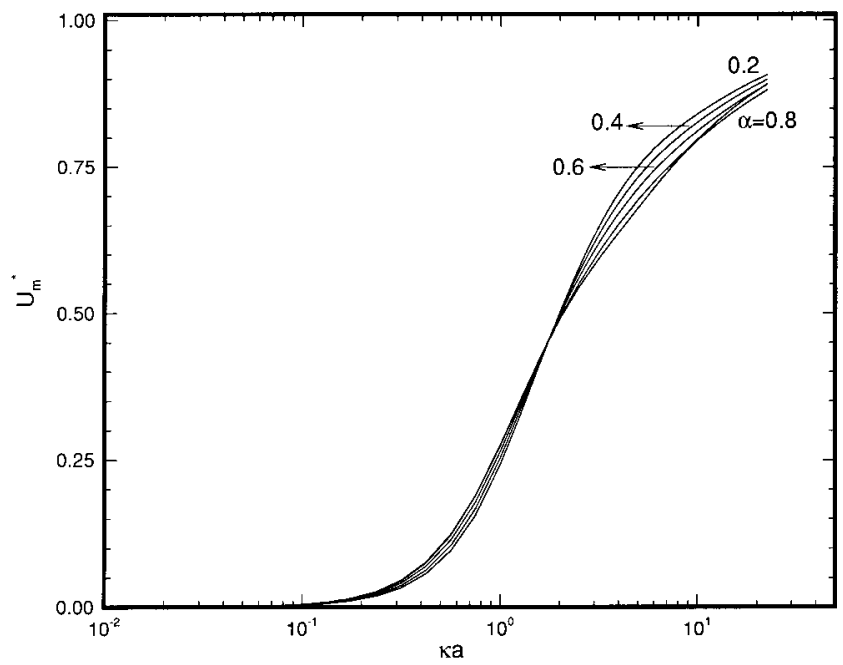

FIG. 10. Variation of scaled mobility $U_{m}^{*}$ as a function of $\kappa a$ at various values of $\alpha$ (= valence of anions/valence of cations) for the case in Fig. 9. 


\section{ACKN OWLED GMENT}

This work is supported by the National Science Council of the Republic of China.

\section{REFERENCES}

1. Von Smoluchowski, M., Z. Phys. Chem. 92, 129 (1918).

2. Huckel, E., Phys. Z. 25, 204 (1924).

3. Henry, D. C., Proc. R. Soc. London Ser. A 133, 106 (1931).

4. Booth, F., Proc. R. Soc. London Ser. A 203, 514 (1950).

5. Wiersema, P. H., Loeb, A. L., and Overbeek, J. Th. G., J. Colloid Interface Sci. 22, 78 (1966).

6. O'Brien, R. W., and White, L. R., J. Chem. Soc. Faraday Trans. 2 74, 1607 (1978).

7. O’Brien, R. W., and Hunter, R. J., Can. J. Chem. 59, 1878 (1981).
8. Ohshima, H., Healy, T. W., and White, L. R., J. Chem. Soc. Faraday Trans. 2 79, 1613 (1983).

9. Ohshima, H., Adv. Colloid Interface Sci. 62, 189 (1995).

10. Levine, S., and Neale, G. H., J. Colloid Interface Sci. 47, 520 (1974).

11. Kozak, M. W., and Davis, E. J., J. Colloid Interface Sci. 127, 497 (1989).

12. Kozak, M. W., and Davis, E. J., J. Colloid Interface Sci. 129, 166 (1989).

13. Ohshima, H., J. Colloid Interface Sci. 188, 481 (1997).

14. Zydney, A. L., J. Colloid Interface Sci. 169, 476 (1995).

15. Lee, E., Chu, J. W., and Hsu, J. P., J. Colloid Interface Sci. 196, 316 (1997).

16. Lee, E., Chu, J. W., and Hsu, J. P., J. Colloid Interface Sci. 205, 65 (1998). 